\title{
Structural analysis of the DNA-binding domain of alternatively spliced steroid receptors
}

\author{
L Wickert and J Selbig ${ }^{\mathbf{1}}$ \\ Department of Clinical Biochemistry, Medical Center, University of Bonn, Sigmund-Freud-Strasse 25, D-53105 Bonn, Germany \\ ${ }^{1}$ Institute for Algorithms and Scientific Computing (SCAI) GMD, German National Research Center for Information Technology, Schloss Birlinghoven, \\ D-53754 Sankt Augustin, Germany \\ (Requests for offprints should be addressed to L Wickert, Institute of Clinical Chemistry and Pathobiochemistry, RWTH-University Hospital Aachen, \\ Pauwelsstr. 30, D-52074 Aachen, Germany; Email: wickert@unicum.de) \\ (J Selbig is now at Max Planck Institute of Molecular Plant Physiology, Am Mühlenberg 1, 14476 Golm, Germany)
}

\begin{abstract}
We have generated 24 DNA-binding domain structure models of alternatively spliced or mutated steroid receptor variants by homology-based modeling. Members of the steroid receptor family dispose of a DNA-binding domain which is built from two zinc fingers with a preserved sequence homology of about $90 \%$. Data from crystallographic analysis of the glucocorticoid receptor DNAbinding domain are therefore appropriate to serve as a template structure. We inserted or deleted amino acid residues in order to study the structural details of the glucocorticoid, mineralocorticoid, and androgen receptor splice variants. The receptor variants are created by QUANTA- and MODELLER-based modeling. Subsequently, the resulting energy-minimized models were compared with each other and with the wild-type receptor respectively. A prediction for the receptor function based mainly on the preservation or destruction of secondary structures has been carried out. The simulations
\end{abstract}

showed that amino acid insertions of one, four or nine additional residues of existing steroid receptor splice variants were tolerated without destruction of the secondary structure. In contrast, a deletion of four amino acids at the splice site junction leads to modifications in the secondary structure of the DNA-recognition helix which apparently disturb the receptor-DNA interaction. Furthermore, an insertion of 23 amino acid residues between the zinc finger of the androgen receptor leads to a large loop with an additional $\alpha$-helical structure which seems to disconnect a specific contact from its hormone response element. Thereafter, the prediction of receptor function based on the molecular models was compared with the available experimental results from the in vitro function tests. We obtained a close correspondence between the molecular modeling-based predictions and the conclusions of receptor function drawn from in vitro studies.

Journal of Endocrinology (2002) 173, 429-436

\section{Introduction}

The members of the steroid receptor family (glucocorticoid, mineralocorticoid, progesterone, androgen and estrogen receptors) act as ligand-dependent transcription factors. All receptors are provided with a highly conserved DNA-binding domain (DBD) consisting of two zinc fingers each, which are expressed by separate exons (Evans 1988). One zinc finger establishes the receptor-DNA contact by binding a specific hormone response element (HRE), the other is involved in receptor dimerization (Fig. 1). For example, the important amino acid residues responsible for the DNA-receptor interaction in the wild-type glucocorticoid receptor (GR) are localized in the P-box of the first zinc finger (C457-C460) and in the interfinger region (V462-Y474). Near the P-box, a transition in the secondary structure from a $\beta$-strand (C450L455) to an $\alpha$-helix (G458-E469) can be observed; this was termed the DNA-recognition helix by Luisi et al. (1991). As well as the adjacent exon junction (exon 3 (G470) to exon 4 (Q471)), the D-box of the second zinc finger up to the amino acid D485 builds an extended coil, which is followed by a second helix (P493-L504). The molecular shape of the zinc fingers is determined by the tetrameric co-ordinative binding of four cysteins with a central $\mathrm{Zn}^{2+}$ ion (Luisi et al. 1991). Thus, the conserved amino acid sequence and the resulting $3 \mathrm{D}$ structure provide for an optimal, specific DNA binding and are a prerequisite for regulating target gene expression by steroid hormone receptors.

Alternative splicing of exons 3 and 4 of the mineralocorticoid receptor (MR) and GR leads to mRNAs with an additional in-frame insertion in the interfinger region between the two zinc fingers of the DBD (Fig. 1). Here, the alternative used donor splice site at the end of exon 3/intron $\mathrm{C}$ rejoins with the conserved acceptor splice 


\section{DBD}
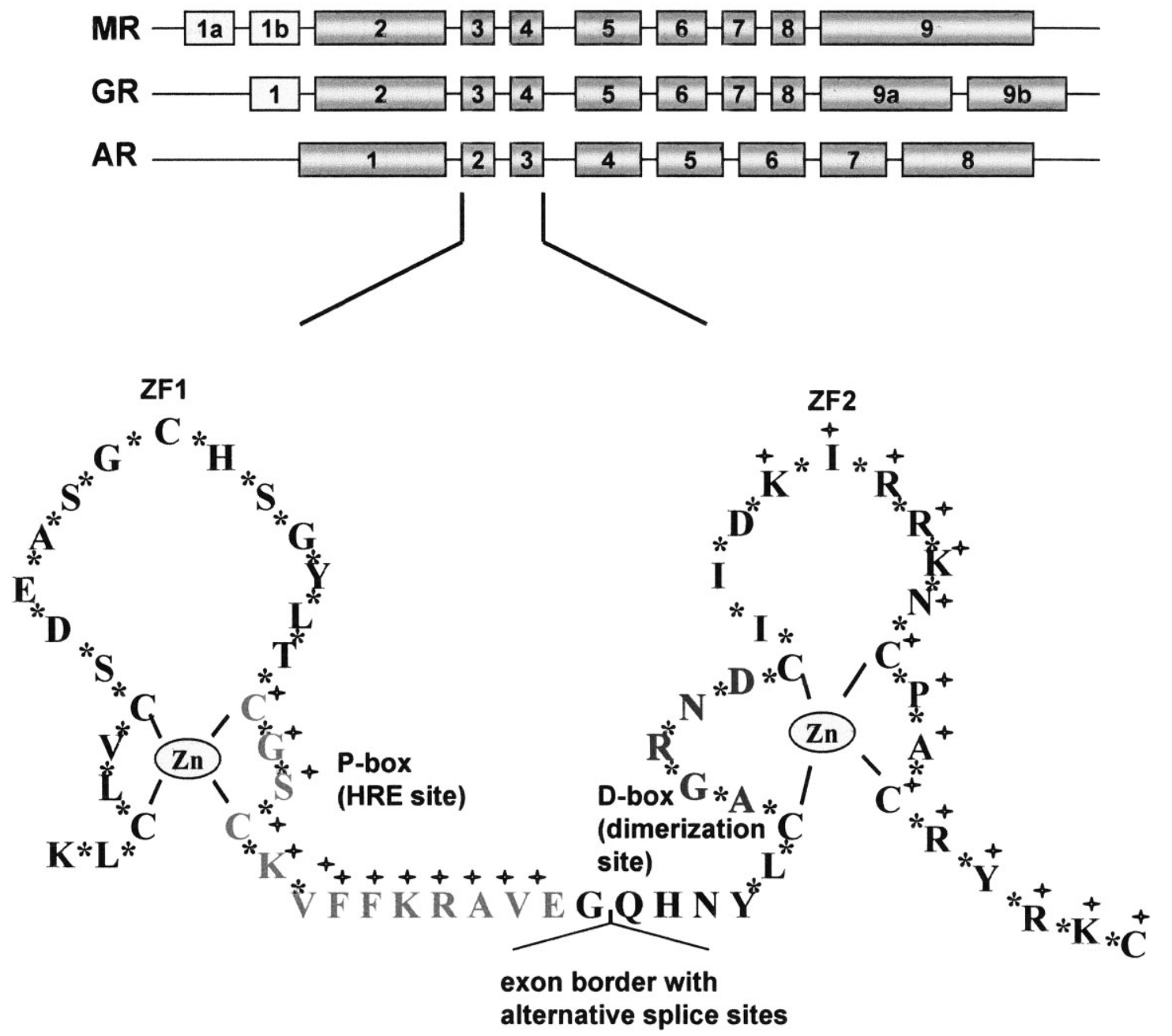

Figure 1 Organization and structure of the DNA binding domain of the MR, GR and AR genes. White boxes, untranslated exons; grey boxes, translated exons. Amino acid sequence in one letter code: grey letters, DNA identification sequence. Amino acids marked with a plus sign form $\alpha$ helices. ZF, zinc finger.

site of intron C/exon 4 (Bloem et al. 1995). Corticoid receptors (MR and GR) from different species with an insertion of one $(\mathrm{GR}+1$ in primate), four $(\mathrm{MR}+4$ in human, rat and Xenopus laevis) or nine (GR +9 in fish) additional amino acid residues were described by several groups (Brandon et al. 1991, Bloem et al. 1995, Csikos et al. 1995, Ducouret et al. 1995, Tokuda 1998, Wickert et al. 1998). Surprisingly, despite the extension of nine amino acids between the two zinc fingers, the fish GR +9 showed transcriptional activity in a luciferase reporter gene assay (Ducouret et al. 1995). Due to a computer-based reconstruction of the phylogenetic tree, these authors suggested that the GR +9 splice variant appears to be the most ancient receptor of the GR group. However it remains obscure how the receptor accomplishes the binding to the glucocorticoid response element (GRE) which was used in the reporter gene assay (Ducouret et al. 1995).

The interfinger region of the GR DBD seems to be flexible and can cope with insertions of up to 27 amino acid residues according to Zandi et al. (1993). A receptor fragment consisting of its $\mathrm{N}$-terminal domain and a partial duplication of the second zinc finger in the DBD showed a limited activity of 10-20\% when compared with the wild-type receptor in a transactivation test (Zandi et al. 1993).

Moreover, an androgen receptor (AR) with 23 additional amino acid residues $(A R+23)$ which were inserted at the splice site junction of the zinc finger coding exons 2 and 3 has been found in a family with partial 
androgen insensitivity (Brüggenwirth et al. 1997). In this particular case, the acceptor splice site was used alternatively and 69 base pairs of the intronic sequence, upstream from exon 3, were inserted. The additional residues between the two zinc fingers of the $A R+23$ lead to a non-functioning receptor protein in vivo and in vitro (Brüggenwirth et al. 1997). A gel retardation assay showed that the $A R+23$ splice variant was unable to contact the corresponding DNA sequence (androgen response element) in vitro.

To verify the structural changes in the interfinger region of the glucocorticoid, mineralocorticoid, and androgen receptor splice variants we used computer-based modeling by homology. In this study 24 DBD receptor models were generated using the QUANTA Protein Design tool (Molecular Simulations, Inc. 1997) and/or the MODELLER program (Šali \& Blundell 1993) and were compared with the GR wild-type (wt) zinc finger model. Following our structure prediction the hinge region of the zinc finger coding exons of the GR appears to be flexible due to the insertion of several amino acids (up to nine were tested). However, a deletion of four residues upstream or downstream from the exon junction shortened the DNA-recognition helix by about one and a half helical turns. Furthermore, all AR +23 models created by MODELLER (five models per sequence) showed an additional $\alpha$-helix. Two predicted models according to the sequence AR + 23 (GDB M27425) have intimate contact with the DNA molecule, the inserted amino acid residues of one model had only a slight DNA contact, and in two cases the inserted amino acid residues were distorted towards the outside of the protein. The other AR +23 models obtained from sequences GDB M35846 and GDB120556 (see below) resulted in no appreciable difference to the models above. Moreover, the comparison between our prediction of receptor function correlated well with the available transactivation tests.

\section{Materials and Methods}

\section{Sequence alignment and secondary structure prediction}

Sequence-sequence alignments were computed using standard dynamic programming-based alignment methods. A database search for homologous nucleic acid and amino acid sequences was carried out with BLAST (Altschul et al. 1997) over SWISS-PROT (Bairoch \& Apweiler 1997). The following sequences were aligned: (i) AVEGWRAR QNTDGQHNY, the inserted nine amino acids (GR +9) with the adjacent four residues (adjacent residues, AVEG and QHNY were involved, since these are conserved amino acid residues of this receptor family); (ii) the inserted $69 \mathrm{bp}$ nucleotide sequence from $\mathrm{AR}+23$ using data from the Genome Databank (GDB): GDB M27425 (Lubhahn et al. 1989), GBD M35846 (Tilley et al. 1989, Marcelli et al. 1990) and GDB 120556 (Brüggenwirth et al. 1997) respectively; (iii) the corresponding translated amino acid sequence consisting of 23 residues, forward as well as reverse respectively. Secondary structure prediction has been carried out with $\mathrm{CoDe}$, a method combining the results of well-known approaches such as PHD, Predator, and DSC (Rost \& Sander 1993, Frishman \& Argos 1996, King \& Sternberg 1996) to a consensus secondary structure prediction. CoDe is integrated into the ToPLign system (Mevissen et al. 1994, Selbig et al. 1999).

\section{Molecular modeling}

For homology-based modeling the crystallographic analysis of the GR DBD published by Luisi et al. (1991) (protein data bank (PDB) entry: 1 glu; PDB (Bernstein et al. 1977)) and the GR NMR-structure published by Bauman et al. (1993) (PDB entry: 2 gda (24 structures) or 1 gdc (minimized average structure)) were used. The GR DBD sequence was edited using the QUANTA Protein Design tool. The CHARMm force field-based energy minimization was initially performed with the steepest descent algorithm and finalized using the conjugate gradient method. In general, energy minimization calculations as part of homology modeling are performed with parts of the molecule held fixed. This is necessary because alterations of the structure induced by minimization are dragged towards lowering the energy. Structures of low potential energy are useless if they come with poor geometry. For instance, in the case of substituting amino acids, only the energy of the modeled protein side chain should be minimized. The situation is more complicated in cases where several amino acids are deleted or inserted. For this type of alterations there are no coordinates of backbone atoms which can be copied from the template. Therefore, larger regions of the protein have to be set free for minimization. Because we were interested, mainly, in whether secondary structures are preserved or destroyed, we tested several variable residue ranges extending in both the $\mathrm{N}$ - as well as the C-terminal direction from the insertion or deletion site (plus/minus 1, 4 and 20 amino acids). The following protein models were subjected to the simulations: human (h) GRwt, marmoset (mt) GR+1, Onchorhynchus mykiss (om) GR +9, hGR-G, hGR-Q, hGR-AVEG, hGR-QHNY, MRwt, MR + 4, as receptor monomers.

For modeling the $\mathrm{AR}+23$ structure a different protocol had to be applied. Currently, there is no modeling tool that could model segments reliably whose length exceeds 10-12 residues (A Fiser \& A Šali, personal communication). A database search of the 23 residue fragment with BLAST against the PDB structures provided the homeodomain of the thyroid transcription factor 1 (PDB entry: $1 \mathrm{ftt})$ as a possible template. Thus, together with the GR $\mathrm{DBD}$ as a template for the basic AR receptor structure, a hybrid model could be derived with the MODELLER program. According to the AR cDNA sequence (Tilley 
Table 1 Multiple alignment of different steroid receptors which are alternatively spliced in their DBD. Sequence alignments of the steroid receptor splice variant DBD models. Secondary structures according to the QUANTA assignment are shown in the following colored letters: green=beta bulge, yellow $=\mathrm{H}$ bonded $\beta$-strand, violet $=\alpha$-helix, blue $=3$ er turn, red $=4$ turn, black $=$ irregular structure

\begin{tabular}{|c|c|c|}
\hline \multirow[b]{2}{*}{ Receptor } & \multicolumn{2}{|l|}{ Sequence } \\
\hline & $\begin{array}{ll}\text { zinc finger } 1 & \text { exon junction } \\
\text { P-Box } & \end{array}$ & $\begin{array}{c}\text { zinc finger } 2 \\
\text { D-box }\end{array}$ \\
\hline hGRwt, hMRwt (1) & LTCGSCKVFFKRAVEG & QHNYLCAGRNDCIIDKIR \\
\hline $\mathrm{hMR}+4, \mathrm{rMR}+4(2,3)$ & LTCGSCKVFFKRAVEG & KCSWQHNYLCAGRNDCIIDKIR \\
\hline $\mathrm{xIMR}+4(4)$ & LTCGSCKVFFKRAVEG & KCSRQHNYLCAGRNDCIIDKIR \\
\hline $\mathrm{GR}+1(5)$ & LTCGSCKVFFKRAVEG & RQHNYLCAGRNDCIIDKIR \\
\hline om, poGR+9 $(6,7)$ & LTCGSCKVFFKRAVEG & WRARQNTDGQHNYLCAGRNDCI IDKIR \\
\hline hGR exon 3 del G (8) & LTCGSCKVFFKRAVE & QHNYLCAGRNDCIIDKIR \\
\hline hGR exon 4 del Q (8) & LTCGSCKVFFKRAVEG & HNYLCAGRNDCIIDKIR \\
\hline hGR exon 3 del AVEG (8) & LTCGSCKVFFKR & QHNYLCAGRNDCIIDKIR \\
\hline hGR exon 4 del QHNY (8) & LTCGSCKVFFKRAVEG & LCAGRNDCIIDKIR \\
\hline hARwt & LTCGSCKVFFKRAAEG & KQKYLCASRNDCTIDKIR \\
\hline hAR + $23($ GDB M27425) (9) & LTCGSCKVFFKRAAEGKYPKKETLET & VYQLLYLFSQKQKKYLCASRNDCTIDKIR \\
\hline$h A R+23($ GDB M35846) (9) & LTCGSCKVFFKRAAEGKYPKKETLET & VNQLLFVFSQKQKYLCASRNDCTIDKIR \\
\hline $\mathrm{hAR}+23(\mathrm{GDB} 120556)(9)$ & LTCGSCKVFFKRAAEGKYPKKETLET] & VYQLLFVFSQKQKYLCASRNDCTIDKIR \\
\hline
\end{tabular}

h, human; r, rat; $\mathrm{xl}$, Xenopus laevis (Anura, amphibia); mt, marmoset (Callitrichidae, new world monkey); om, Onchorhynchus mykiss (Teleostei, fish); po, Paralichtys olivaceus (Actinopterygii, fish); wt, wild type; del, deletion. (1) Luisi et al. (1991); (2) Bloem et al. (1995); (3) Wickert et al. (1998); (4) Csikos et al. (1995); (5) Brandon et al. (1991); (6) Ducouret et al. (1995); (7) Tokuda (1998); (8) Zandi et al. (1993); (9) Brüggenwirth et al. (1997).

et al. 1989) the following amino acids were substituted: V468 to A; Q471, H472, N473 to K, Q, and K respectively; G478 to $\mathrm{S}$ and $\mathrm{I} 483$ to T. Sequence specification of human AR intron 2/exon 3 junction varied. Hence, 15 hAR +23 hybrid models were generated according to the sequences from GDB M27425, M35846 and 120556 respectively.

\section{Results and Discussion}

Utilizing the zinc finger homology of the steroid hormone receptors we generated 24 computer-based protein models with the QUANTA Protein Design tool and MODELLER by using the data of the crystallographic analysis of the GR DBD (Luisi et al. 1991). The colored alignment in Table 1 indicates the changes in the secondary structure of the different steroid receptor DBD models.

Human, rat and Xenopus laevis $M R+4(h M R+4$, $r M R+4, x(M R+4)$

The published $\mathrm{hMR}+4 \mathrm{DBD}$ model with the inserted amino acids KCSW contains a small loop which was distorted to outside the protein (Wickert et al. 2000). In our study a new model was created with a transposition of $\mathrm{W}$ to $\mathrm{R}$. The superposition of both energy-minimized $\mathrm{MR}+4$ models shows no appreciable difference in the loop shape and orientation. Interestingly, the arginine ( $R$ ) residue of the $\mathrm{xlMR}+4$ model oriented towards the tryptophan (W) residue of the hMR +4 model in a perpendicular manner. Apparently, in this case it appears to be of no importance whether the inserted amino acid sequence is KCSW (human and rat) or KCSR (Xenopus laevis).

Marmoset GR +1 and Onchorhynchus mykiss GR +9 $(m t G R+1, o m G R+9)$

The insertion of an arginine in the marmoset GR $(G R+1)$ occurs at a position which coincides with single amino acid insertions in the human AR (K405) and the V-erb-A and C-erb-A protooncogenes (N68 and N82 respectively) (Brandon et al. 1991). According to our model, this additional residue allows for a new hydrogen bond between the oxygen of the glycine (G470) and the hydrogen of the histidine (H472) of the primate GR +1 . Therefore, a new 3-turn at the exon junction was formed (Table 1, blue) between the arginine (inserted residue) and the glutamine (Q471). The modified secondary structure in the GR +1 model would support the hypothesis of Brandon and colleagues (1991), who suspected a change in the affinity of receptor-DNA interaction.

Surprisingly, another GR splice variant was described by Ducouret and co-workers (1995). Here, nine additional amino acid residues (WRARQNTDG) were inserted in the interfinger region of the GR DBD of the rainbow trout Onchorhynchus mykiss. Transfection studies showed that the GR +9 binds mammalian GRE and is able to activate a reporter gene. As with the $\mathrm{MR}+4$ model 


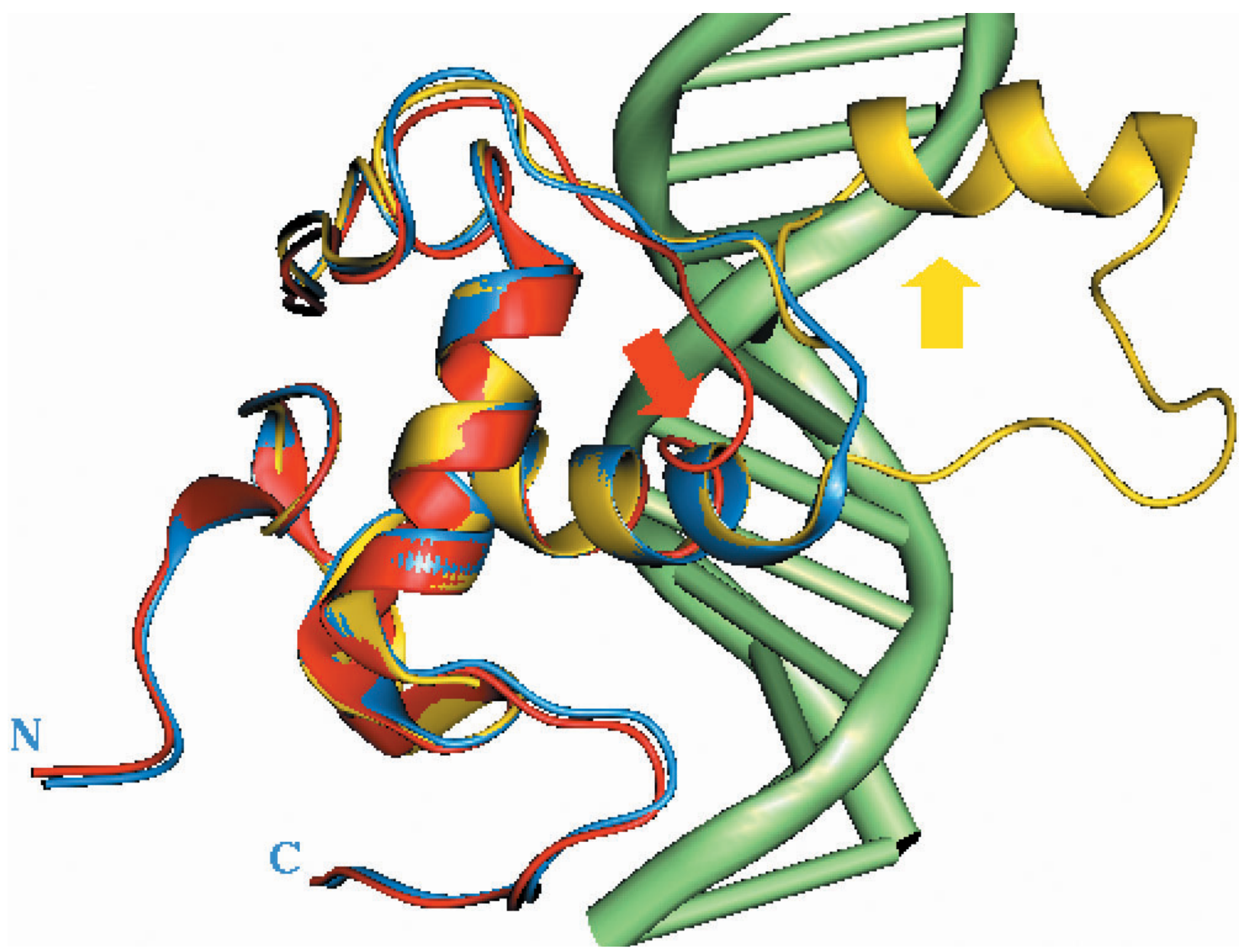

Figure 2 Superposition of the generated GRwt, GR-QHNY and AR+23 DBD models. Crystal structure data for computer-based modeling by homology were obtained from GR (Luisi et al. 1991) (PDB 1 glu). Here, only chain A monomeres of GRwt (blue), GR-QHNY (red) and AR+23 (yellow) were superimposed. The red arrow shows the shortening of the DNA-recognition helix in the GR-QHNY model. The additional helical structure formed by the inserted amino acid residues of the AR+23 model is marked by a yellow arrow. The ribbons indicate $\alpha$-helices which are regular regions of the protein fragments. The threads are non-regular regions. DNA is shown as a double-helix ladder with rungs in metallic green.

(Wickert et al. 2000), the GR +9 model emulated a loop structure distorted to outside the protein.

Notably, in all generated corticoid receptor DBD models with an insertion of one, four or nine amino acid residues, the glutamic acid (E469) was excluded from forming the DNA-recognition helix (Table 1).

\section{Human GR-G, GR-Q, GR-AVEG and GR-QHNY}

In 1993 Zandi and colleagues noted that no functional GR with a deletion in the interfinger region could be obtained. In their opinion a shortening of the interfinger region would force the zinc finger into a different, unsuitable angle. This would disrupt the hydrophobic core which is formed in the wild-type receptor between some residues of the DNA-recognition helix and the following helix in the second zinc finger (C493-G504). Apparently, the hydrophobic core seems to play an important role in the mutual position of the two zinc fingers (Zandi et al. 1993). To verify this hypothesis, we generated GR DBD models with several deletion mutations at the exon junction between exons 3 and 4 . One or four amino acid residues were deleted from the donor splice site as well as from the acceptor splice site, GR-G, GR-Q, GR-AVEG and GR-QHNY respectively. Superposition of the GR-G and GR-Q resulted in nearly congruent models and both showed a slight deviation when compared with the wild-type receptor model.

The deletion of four amino acid residues in the DBD results in larger conformational changes in the interfinger region. As the wild-type receptor model showed a helical conformation in the interfinger region from the amino 
Table 2 Secondary structure prediction of the 23 amino acids inserted into a human androgen receptor splice variant. Comparison of the results of selected secondary structure prediction methods used by the consensus prediction program CoDe with the secondary structure of the template for the 23 inserted amino acids

\author{
23 Inserted amino acids \\ Sequence fragment $1 \mathrm{ftt}$ \\ DSSP assignment \\ Prediction program \\ PHD \\ Predator \\ DSC \\ CoDe consensus
}

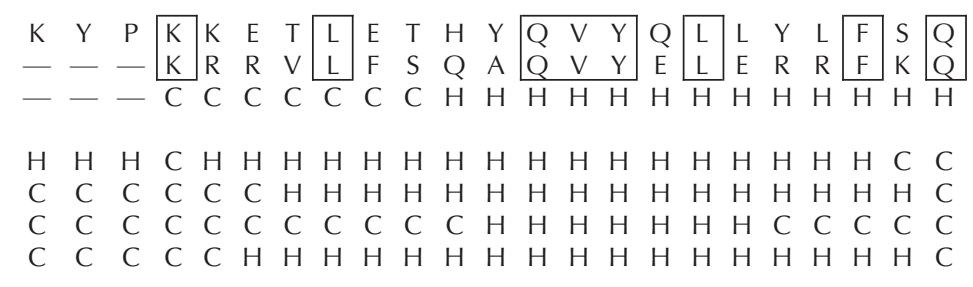

Amino acid sequences in one-letter code; $1 \mathrm{ftt}$, thyroid transcription factor 1 ; DSSP, database of secondary structure in proteins (Kabsch \& Sander 1983); C, coil; H, $\alpha$-helix. The boxes indicate identical residues. acid residues $\mathrm{G} 458$ to $\mathrm{E} 469$, the $\alpha$-helical structure in the deletion mutant models GR-AVEG and GR-QHNY were damaged (Table 1, Fig. 2). Both models showed a shortening in the interfinger helix of about six residues that corresponds to one and a half helical turns. The amino acids FKRA (464-467) of the GR-QHNY model build an apparent 5-turn structure, which is really composed of two overlapping 3-turn structures (Fig. 2, red arrow). Our structure prediction of the deletion splice variants supports the hypothesis of Zandi and co-workers (1993) which states that the mutual distance of the two zinc fingers is changed. One consequence might be that these receptors are unable to bind the hormone response element in the promotor region of the target gene. It is known from gene-targeting studies with disrupted glucocorticoid receptors in mouse that a failing GR leads to death within the first hours after birth (Berger et al. 1996). This might be the reason why no GR with a deletion in the interfinger region was discovered.

\section{Human ARwt and AR+23}

The BLAST-based comparison of the 23 amino acid residues with sequences of known structures from the PDB resulted in a potential relatedness between this fragment and the homeobox domain of the thyroid transcription factor 1 . Interestingly, a corresponding search in several sequence databases did not produce this hit. This may result from the fact that the alignment values depend on the size of the database against which a target sequence is compared. The potential relatedness is supported by the secondary structure of the 23 amino acid fragment predicted with CoDe (Selbig et al. 1999) (Table 2). The structural fragment from the thyroid transcription factor 1 (PDB: $1 \mathrm{ftt}$ ) together with the GR DNA-binding domain (PDB entry: 1 glu) allowed us to generate a hybrid model for $A R+23$. For each of the three observed human $\mathrm{AR}+23$ sequences we derived five models. In all of these models the inserted 23 amino acid residues form a large loop with an adjacent additional $\alpha$-helix varying in length by 1-2 residues. Several of these models feature an intimate contact with the DNA molecule, touching it beside the regular cis-active DNA sequence, which is the HRE (Fig. 2, yellow arrow). The loop structure of another $\mathrm{AR}+23$ model (GDB M27425) has only slight DNA contact and in two cases the loop of the inserted amino acid residues was distorted to the protein outside. Figure 2 illustrates an AR +23 DBD model that provided the best structural explanation for the loss of receptor function. In all fifteen models the side chains of the inserted amino acid residues require so much space that a normal contact with the HRE seems to be impossible. The unusual mutated $A R+23$ caused a partial androgen insensitivity, which can be explained either by a damaged DNA contact through the inserted loop or by a failure in dimerization with the second hormone-receptor complex. The probable loss of the ability of DNA binding is supported by results from a gel-retardation assay obtained from Brüggenwirth et al. (1997).

\section{Prediction by homology-based molecular modeling versus results} obtained from in vitro assays

Furthermore, we compared our prediction of receptor function with the available in vitro transactivation tests on the basis of the steroid receptor models. As mentioned above, both methods correlated highly (Table 3). All steroid receptor splice variant models allow for structural explanations which confirm the experimental findings. Through revised and further developed methods for sequence-structure alignments and the homology-based modeling the reliability of the predicted structures improved (Kolinski et al. 1999, Šali \& Kuriyan 1999, Selbig et al. 1999). Even sequences with a relatively low identity could be used for modeling by homology (Tramontano 1998) and this method could serve as a tool for gene annotation strategies (Francoijs et al. 2000). 

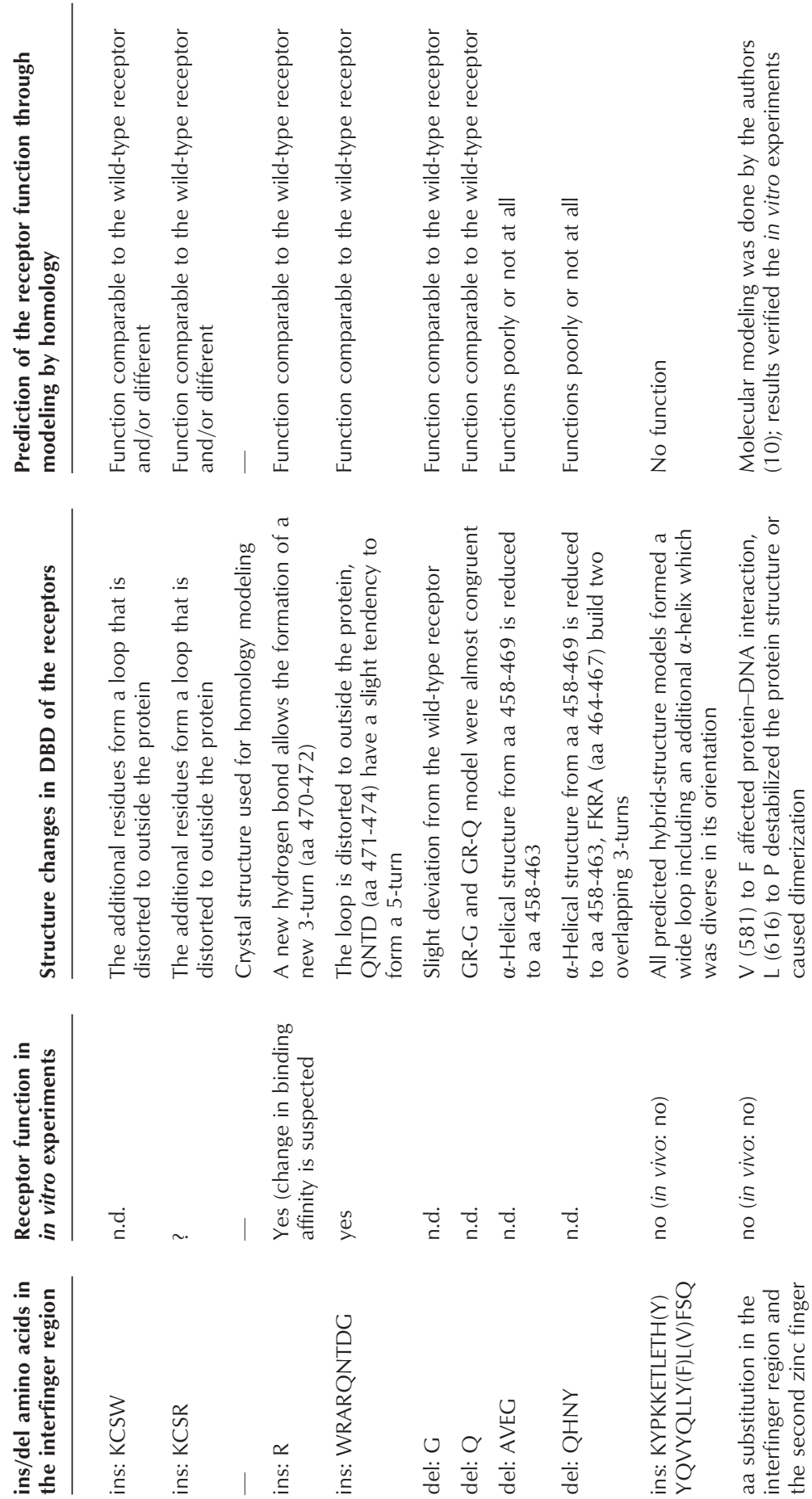

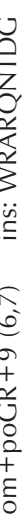

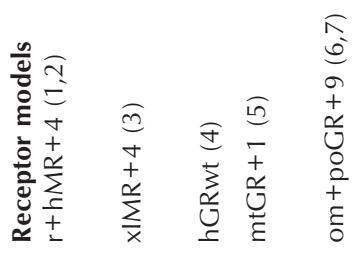

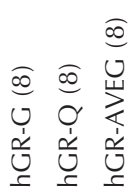

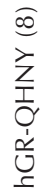

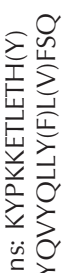

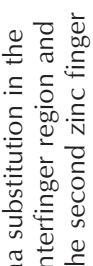

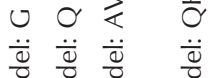

i⿱宀

I. $\cong \pm$ 
Actually, we were not able to generate NMRstructures or crystal structures from every splice variant. Therefore, modeling by homology seems to offer the only possibility of describing and explaining alterations in molecular structures. Future experiments where homology models are generated in advance of functional examinations will show whether functional predictions from homology models are possible.

\section{References}

Altschul SF, Madden TL, Schäffer AA, Zhang J, Zhang Z, Miller W \& Lipman DJ 1997 Gapped BLAST and PSI-BLAST: a new generation of protein database search programs. Nucleic Acids Research 25 3389-3402.

Bairoch A \& Apweiler R 1997 The SWISS-Prot protein sequence database: its relevance to human molecular medical research. Journal of Molecular Medicine 75 312-316.

Bauman H, Paulsen K, Kovacs H, Berglund H, Wrught AP, Gustafsson JA \& Härd T 1993 Refined solution structure of the glucocorticoid receptor DNA-binding domain. Biochemistry $\mathbf{3 2}$ 13463-13471.

Berger S, Cole TJ, Schmid W \& Shutz G 1996 Analysis of glucocorticoid and mineralocorticoid signalling by gene targeting. Endocrinology Research 22 641-652.

Bernstein FC, Koetzle TF, Williams GJB, Meyer EF Jr, Brice MD, Rodgers JR, Kennard O, Shimanouchi T \& Tasumi M 1977 The protein data bank: a computer based archival file for macromolecular structures. Journal of Molecular Biology 112 535-542.

Bloem LJ, Guo C \& Pratt JH 1995 Identification of a splice variant of the rat and human mineralocorticoid receptor genes. Journal of Steroid Biochememistry and Molecular Biology 55 159-162.

Brandon DD, Markwick AJ, Flores M, Dixon K, Albertson BD \& Loriaux DL 1991 Genetic variation of the glucocorticoid receptor from a steroid-resistant primate. Journal of Molecular Endocrinology 7 89-96.

Brüggenwirth HT, Boehmer ALM, Ramnarain S, Verleun-Mooijman MCT, Satijn DPE, Trapman J, Grootegoed JA \& Brinkmann AO 1997 Molecular analysis of an androgen-receptor gene in a family with receptor-positive partial androgen insensitivity: an unusual type of intronic mutation. American Journal of Human Genetics $\mathbf{6 1}$ 1067-1077.

Csikos T, Tay J \& Danielsen M 1995 Cloning and characterization of the Xenopus laevis mineralocorticoid receptor, Accession no U15135 (EMBL), direct submission.

Ducouret B, Tujague M, Ashraf J, Mouchel N, Servel N, Valotaire Y \& Thompson EB 1995 Cloning of a teleost fish glucocorticoid receptor shows that it contains a deoxyribonucleic acid-binding domain different from that of mammals. Endocrinology 136 3774-3783.

Evans RM 1988 The steroid and thyroid hormone receptor superfamily. Science 240 889-895.

Francoijs CJJ, Klomp JPG \& Knegtel RMA 2000 Sequence annotation of nuclear receptor ligand-binding domains by automated homology modeling. Protein Engineering 13 391-394.

Frishman D \& Argos P 1996 Incorporation of non-local interactions in protein secondary prediction from the amino acid sequence. Protein Engineering 9 133-142.

Kabsch W \& Sander C 1983 Dictionary of protein secondary structure: pattern recognition of hydrogen-bonded and geometrical features. Biopolymers 22 2577-2637.
King R \& Sternberg MJ 1996 Identification and application of the concepts important for accurate and reliable protein secondary structure prediction. Protein Science 17 3389-3407.

Kolinski A, Rotkiewicz P, Ilkowski B \& Skolnick J 1999 A method for the improvement of threading-based protein models. Proteins $\mathbf{3 7}$ 592-610.

Lobaccaro JM, Poujol N, Chiche L, Lumbroso S, Brown TR \& Sultan C 1996 Molecular modeling and in vitro investigation of the human androgen receptor DNA-binding domain: application for the study of two mutations. Molecular and Cellular Endocrinology 116 137-147.

Lubahn DB, Brown TR, Simental JA, Higgs HN, Migeon CJ, Wilson EM \& French FS 1989 Sequence of the intron/exon junctions of the coding region of the human androgen receptor gene and identification of a point mutation in a family with complete androgen insensivity. PNAS 86 9534-9538.

Luisi BF, Xu WX, Otwinowski Z, Freedman LP, Yamamoto KR \& Sigler PB 1991 Crystallographic analysis of the interaction of the glucocorticoid receptor with DNA. Nature 352 497-505.

Marcelli M, Tilley WD, Wilson CM, Griffin E, Wilson JD \& McPhaul MJ 1990 Definition of the human androgen receptor gene structure permits the identification of mutations that cause androgen resistance: premature termination of the receptor protein at amino acid residue 588 causes complete androgen resistance. Molecular Endocrinology 4 1105-1116.

Mevissen H, Thiele R, Zimmer R \& Lengauer T 1994 The ToPLign software environment - Toolbox for protein alignment. Bioinformatik, Institut für molekulare Biotechnologie, Jena, http://cartan.gmd.de/ ToPLign.html.

Rost B \& Sander C 1993 Prediction of protein secondary structure at better than 70\% accuracy. Journal of Molecular Biology 232 584-599.

Šali A \& Blundell T 1993 Comparative protein modeling by satisfaction of spatial restraints. Journal of Molcular Biology 239 779-818.

Šali A \& Kuriyan J 1999 Challenges at the frontiers of structural biology. Trends in Biochemical Science 24 M20-M24.

Selbig J, Mevissen T \& Lengauer T 1999 Decision tree-based formation of consensus protein secondary structure prediction. Bioinformatics 15 1039-1046.

Tilley W, Marcelli M, Wilson JD \& McPhaul M 1989 Characterization and expression of a cDNA encoding the human androgen receptor. PNAS 86 327-331.

Tokuda 1998 Accession no O73673 (EMBL), direct submission.

Tramontano A 1998 Homology modeling with low sequence identity. Methods: A Companion to Methods in Enzymology 14 293-300.

Wickert L, Watzka M, Bolkenius U, Bidlingmaier F \& Ludwig M 1998 Mineralocorticoid receptor splice variants in different human tissue. European Journal of Endocrinology 138 702-704.

Wickert L, Selbig J, Watzka M, Stoffel-Wagner B, Schramm J, Bidlingmaier F \& Ludwig M 2000 Differential mRNA expression of the two mineralocorticoid receptor splice variants within the human brain: structure analysis of their different DNA binding domains. Journal of Neuroendocrinology 12 867-873.

Zandi E, Galli I, Döbbeling U \& Rusconi S 1993 Zinc finger mutations that alter domain interactions in the glucocorticoid receptor. Journal of Molecular Biology 230 124-136.

Received 18 February 2002

Accepted 4 March 2002 